\title{
Computerized quantitative radionuclide assessment of heterotopic ossification in spinal cord injury patients
}

\author{
Sun W Kim MD, ${ }^{1}$ Sing Yung Wu MD PhD, ${ }^{2}$ and $\mathrm{R} \mathrm{C} \mathrm{Kim} \mathrm{MD}^{3}$ \\ ${ }^{1}$ Spinal Cord Injury Service (128), Veterans Affairs Medical Center, 130 W. Kingsbridge \\ Road, Bronx, New York 10468, USA; ${ }^{2}$ Nuclear Medicine and Medical Service, \\ ${ }^{3}$ Laboratory Service, Veterans Affairs Medical Center, Long Beach, California, USA.
}

We evaluated the progression of heterotopic ossification (HO) in 17 spinal cord injury patients by comparing radiographs, quantitative radionuclide bone scans, and serum alkaline phosphatase levels. Evidence of maturation of $\mathrm{HO}$ appeared earlier ( 3 months to 6 years post injury) in radiographs, whereas, during the same time frame, radioactive nuclide assessment showed continued progression of $\mathrm{HO}$ in 10 out of the 17 patients. The evolution of $\mathrm{HO}$ appeared to take place over a period ranging between 3 and 80 months. We believe that stabilization of HO may be reasonably defined in terms of uptake ratios of 2.0 or less in patients with initial uptake ratios over 3.0 but below 5.0, and of ratios of 3.0 or less when the initial values are over 5.0.

Keywords: spinal cord injury; heterotopic ossification; radionuclide assessment of heterotopic ossification.

\section{Introduction}

The radionuclide bone scan following neurological injury is capable of detecting heterotopic ossification (HO) earlier than by radiographic means. Initially, the local features of HO may simulate those of deep venous thrombosis or cellulitis. As it is too early to visualize $\mathrm{HO}$ by radiographs at this time, the bone scan is, up to the present time, the only way to establish the diagnosis. It also permits temporal definition of the interval during which $\mathrm{HO}$ stabilizes or matures. This is of particular importance in determining the correct time for operative resection of the mass, because of the potential for postoperative recurrence. ${ }^{1-3}$

Clinically it is of value to be able to assess the progression, maturation, stabilization, and duration of $\mathrm{HO}$ in order to deal with this disturbing complication of spinal cord injury. There are few references in the literature ${ }^{4,5}$ to follow up studies of the progression of $\mathrm{HO}$. The evaluation of bone scan findings has heretofore been of a qualitative rather than of a quantitative nature. The purpose of this report is to provide a quantitative evaluation of the progression of $\mathrm{HO}$ in a series of spinal cord patients.

\section{Material and methods}

Seventeen traumatic myelopathy patients with newly developed $\mathrm{HO}$, all males, were studied. They ranged in age from 28 to 68 (average 40.3) years. Thirteen were white and 4 black. The level of injury was cervical in 13 and thoracic in 4 . The postinjury interval before there was recognition of $\mathrm{HO}$ was less than one month in 3 patients, $1-2$ months in 6, 2-3 months in 4, 3-6 months in 2 , and over 6 months in 2 . All patients were treated with etidronate sodium (disodium1-hydroxyethane diphosphonate, EHDP), $20 \mathrm{mg}$ per $\mathrm{kg}$ for 2 weeks, which was then decreased to $10 \mathrm{mg}$ per $\mathrm{kg}$ for 10 weeks. If a high radionuclide uptake persisted, EHDP was maintained until the uptake was considered stabilized.

An attempt was made to grade the $\mathrm{HO}$ according to the following scale: grade 1, minimal involvement with ossification of less than $3 \mathrm{~cm}$ in maximum dimension; grade 2, partial joint capsule involvement, with more than $3 \mathrm{~cm}$ but less than $7 \mathrm{~cm}$ of ossification; grade 3 , involvement of most of the joint capsule with partial ankylosis of the joint, and more than $7 \mathrm{~cm}$ but less than $15 \mathrm{~cm}$ of ossification; grade 4, complete involvement of the joint capsule, with total 
ankylosis and more than $15 \mathrm{~cm}$ of ossification.

Routine hip radiographs were taken on admission. As soon as the radiographs demonstrated $\mathrm{HO}$ by periodical check every 3 weeks, a computerized bone scan was carried out, except in one patient (case \#15) who never showed $\mathrm{HO}$ radiographically. In this latter patient, a bone scan was performed because of the presence of a mass with swelling, which raised the suspicion of impending HO. Interestingly, bone scans in some individuals disclosed the presence of $\mathrm{HO}$ at additional sites, eg the knees, thighs, and shoulders; these findings were subsequently confirmed radiographically. Approximately 3 hours following the iv administration of 20 millicuries of $99 \mathrm{~m}$-technetium methylene diphosphonate (Tc99mMDP), a total-body PHO/CON bone scan was obtained from the head to below the knees. When $\mathrm{HO}$ was identified, additional views were obtained with an LFOV gamma camera with a parallel-hole collimator. The camera scintigraphic digital 5-minute images, either in an anterior or in a posterior projection, were stored on a computer (MDS A2 with a 80-megabyte disk) in a $128 \times 128$ matrix. The patients were placed in a supine or prone position with the camera oriented perpendicular to the horizontal plane of the scanning table. Extreme care was taken to avoid obliquity. In the digital images, the regions of interest (ROIs) were placed visually over the area of HO. An identical ROI was placed over the uninvolved contralateral joint. When both joints were involved, a third area without bony (background) activity was used. The background regions chosen were either in the paralumbar compartment below the kidneys or in the parafemoral region in the thigh. At these sites, background activity is derived entirely from nonosseous tissue (mainly muscle and skin), and we assumed there would be no significant changes in muscle mass or skin thickness during the course of the interval studies. To ensure reproducibility, digital images were recorded over the same period (160$200 \mathrm{~min}$ ) after Tc99m-MDP injection, and in the same projection. Total counts were obtained either over the ROI or the back- ground region. Serial quantitative determinations were made of the ratios of counts over affected joints and to those of control regions. Preliminary studies showed these ratios to be highly reproducible, with the mean coefficient of variation $(\mathrm{CV})$ in 4 consecutive measurements in each of 2 patients, repositioned when each 5-minute image was taken, being $7.8 \%$ and $8.2 \%$ respectively.

All radiograph and bone scan results were evaluated by the same individual. Follow up radiographs were undertaken at one-month intervals and bone scans were repeated every 6 months unless rapid changes seemed to be taking place, in which case the intervals were shortened. Patients who were discharged before the bone scan results showed decreased uptake or who had evidence of hepatobiliary disease were excluded from the study.

Stabilization of $\mathrm{HO}$ was defined as a combination of the following: (1) distinctness of the outline of the $\mathrm{HO}$ in radiographs; (2) maximum increase in the radiographic density of the HO; (3) lack of further extension of $\mathrm{HO}$ radiographically; and (4) a steady decrease, on more than 2 successive determinations, of radionuclide uptake, with ratios below 2.0 if it was initially over 3.0 but below 5.0, and below 3.0 if it was initially over 5.0. These ratios were selected empirically and arbitrarily on the basis of a preliminary overview of the values obtained. This being a small series, no attempt was made at a statistical comparison of different cutoff points. These ratios, therefore, should be applied only in conjunction with the presence of other criteria, as outlined above.

Serum alkaline phosphatase (AP) levels were measured at 1- to 2-week intervals by the Bessey-Lowry-Brock method, as automated by Morgenstern et $a l ;{ }^{6}$ normal values were considered to be between 30 and 115 mu per ml.

\section{Results}

$\mathrm{HO}$ was found in 30 sites in 17 patients, as is shown in Table I. The number of sites involved was one in 11,2 in 6,3 in 1 , and 4 in 1 . In 10 of the 17 patients, radiographs 
Table I Sites of HO (30 joints in 17 patients)

\begin{tabular}{lccccc}
\hline & Hips & Knees & Thighs & Shoulders & Total \\
\hline Left & 4 & 1 & 1 & 2 & 8 \\
Right & 3 & 0 & 1 & 0 & 4 \\
Both & 8 & 1 & 0 & 0 & 30 \\
Total & 23 & 3 & 2 & 2 & \\
\hline Single joint involvement & & & \\
2 joints & 6 patients & & \\
3 joints & 1 patients & & \\
4 joints & 1 patient & & \\
\hline
\end{tabular}

showed stabilization $\mathrm{HO}$ earlier than did bone scans, as is shown in Table II. Of these 10 patients, 4 showed bone scan findings delayed up to 3 months, 3 from 3 to 6 months, and 3 more than 12 months. These

Table II Stabilization of $\mathrm{HO}$ as seen radiographically and by bone scan

Radiographs earlier than bone scan 10 patients Bone scan earlier than radiographs 3 patients Both simultaneously 4 patients observations indicate that the progression of $\mathrm{HO}$ occurred over a period that lasted up to 3 months in 5 patients, from 3 to 6 months in 4 , from 6 to 12 months in 3 , and over 12 months in 5 (Table III). Quantitative maximum radionuclide uptake ratios varied as is shown in Table III: 3.5 to 7.22 maximum, 1.17 to 2.74 minimum in grade $4 \mathrm{HO} ; 2.87$ to 3.35 maximum, 1.16 to 2.44 minimum in grade $3 ; 1.51$ to 3.88 maximum, 1.05 to 1.77 minimum in grade 2; and 2.04 maximum, 1.10 minimum in grade 1.

Elevation of serum AP correlated with

Table III Duration of HO progression, HO grade, and radionuclide uptake ratios

\begin{tabular}{|c|c|c|c|c|}
\hline Patients & $\begin{array}{l}\text { Duration of } \mathrm{HO} \\
\text { progression }\end{array}$ & $\begin{array}{l}\text { Delay in maturation shown by } \\
\text { bone scan compared to radio- } \\
\text { graphs }\end{array}$ & Grade of $\mathrm{HO}$ & Max/Min uptake \\
\hline$\neq 1$ & 4 mos* & $4 \mathrm{mos}$ & $2 / 1^{* * *}$ & $1.70 / 1.19$ \\
\hline$\neq 2$ & 6 yrs $8 \mathrm{mos}$ & $6 \mathrm{yrs} 1 \mathrm{mo}$ & 4 & $7.03 / 3.68$ \\
\hline$\neq 3$ & 2 yrs $8 \mathrm{mos}$ & 1 yr 3 mos & 4 & $7.22 / 2.87$ \\
\hline$\neq 4$ & 5 yrs $6 \mathrm{mos}$ & simultaneous & 4 & $5.10 / 2.84$ \\
\hline$\neq 5$ & $10 \mathrm{mos}$ & scan 7 mos earlier & 3 & $2.92 / 1.40$ \\
\hline$\neq 6$ & $2 \mathrm{yrs}$ & simultaneous & 3 & $2.87 / 1.16$ \\
\hline$\neq 7$ & 6 yrs 4 mos & 4 yrs & 1 & $1.94 / 1.10$ \\
\hline$\neq 8$ & $9 \mathrm{mos}$ & simultaneous & $2 / 2 / 2 * * *$ & $2.68 / 1.47$ \\
\hline$\neq 9$ & 1 yr 6 mos & $6 \mathrm{mos}$ & 4 & $3.51 / 1.74$ \\
\hline$\neq 10$ & $5 \mathrm{mos}$ & $3 \mathrm{mos}$ & 2 & $1.51 / 1.16$ \\
\hline$\neq 11$ & $10 \mathrm{mos}$ & $4 \mathrm{mos}$ & 2 & $3.38 / 1.51$ \\
\hline$\neq 12$ & $7 \mathrm{mos}$ & simultaneous & 3 & $3.35 / 2.44$ \\
\hline$\neq 13$ & $13 \mathrm{mos}$ & $3 \mathrm{mos}$ & 4 & $3.70 / 1.17$ \\
\hline$\neq 14$ & $12.5 \mathrm{mos}$ & $2.5 \mathrm{mos}$ & 2 & $2.93 / 1.77$ \\
\hline$\neq 15$ & $3 \operatorname{mos}^{* *}$ & $3 \operatorname{mos}$ & 2 & $2.50 / 1.09$ \\
\hline$\neq 16$ & $1 \mathrm{yrs} 4 \mathrm{mos}$ & scan 6 mos earlier & 2 & $2.93 / 1.05$ \\
\hline$\neq 17$ & $1 \mathrm{yr} 1 \mathrm{mo}$ & scan 1 mos earlier & 2 & $1.52 / 1.23$ \\
\hline
\end{tabular}

\footnotetext{
*No growth was noted since first detection in radiographs.

**HO never shown in radiographs.

***More than one grading indicates the $\mathrm{HO}$ grades for more than one site.
} 
scan results in each of the 4 patients tested, but not with radiographic findings in the one tested. AP elevation also correlated with both scan results and radiographic findings in 8 of the 12 patients tested. No relationship was observed between $\mathrm{HO}$ grade and the duration of $\mathrm{HO}$ progression.

\section{Discussion}

Statistical analysis of $\mathrm{HO}$ with regard to incidence, location, level of injury, completeness of paralysis, etc are published elsewhere. ${ }^{1-4}$ This study focuses on the progression of $\mathrm{HO}$ as determined by correlating radiographic and quantitative radionuclide bone scan findings and AP elevation.

All patients were administered EHDP after $\mathrm{HO}$ was discovered. There are reports in the literature ${ }^{2}$ of continued progression of $\mathrm{HO}$ even with EHDP treatment in a small number of patients. This matter will be the subject of a later report.

In the majority (10 patients out of 17$)$ radiographic evidence of $\mathrm{HO}$ maturation preceded that seen in bone scans, which remained positive with increased uptake for varying periods of time. In only 3 of 17 did bone scan results show stabilization earlier than in radiographs. Tanaka et $a l^{5}$ have emphasized that maturation of $\mathrm{HO}$ must be determined on the basis of bone scan findings in order to arrive at a proper decision regarding the timing of surgical intervention for $\mathrm{HO}$.

Since the progression of HO may differ from one patient to the next, maturation of $\mathrm{HO}$ even as seen in bone scans may have considerable variations, ranging from 3 months to 6 years (average 18.9 months) in our patients. This may be the reason why the $\mathrm{HO}$ grade did not correlate with the duration of evolution of HO. In one of our patients uptake suddenly increased after it had decreased in successive determinations. Such an experience has also been recorded by others, ${ }^{5,7}$ who concluded that, in order to judge $\mathrm{HO}$ to be mature, a continuous decrease of the uptake ratio should be followed by a 'steady-state' over a period covering at least 2 or 3 consecutive monthly examinations. These authors concluded, moreover, that if there is a steady decrease in uptake over several months, then surgery, even before a steady-state is reached, will result in a small risk of recurrence. Stover ${ }^{2}$ observed that the precipitating factors that induce bone growth or new foci of $\mathrm{HO}$ included severe systematic illness, hepatic failure, surgery in the $\mathrm{HO}$ area, infection, trochanteric bursitis, and pressure ulcers.

There are no published computerized bone scan criteria for the diagnosis of stabilized HO. We believe, on the basis of this study, that scan uptake ratios of 2.0 (for cases with an initial uptake ratio of over 3.0, but below 5.0) and 3.0 (for cases with an initial uptake ratio of over 5.0) are reasonable criteria for maturation. These criteria, however, may be difficult to apply when there is grade 1 or $2 \mathrm{HO}$.

One possible critique of this study is that EHDP treatment may affect the natural history of HO. As Finerman et $a l^{8}$ and Stover et l $^{9}$ have stated, EHDP is effective in actually preventing $\mathrm{HO}$ formation. It has a high affinity for the calcium ion of hydroxapatite, inhibiting its crystalline growth. All patients in our study, however, were given EHDP after HO was found. The manner in which EHDP affects the uptake ratios is not known.

We do not believe that serum AP levels are reliable indicators of $\mathrm{HO}$. As we have reported recently, ${ }^{10}$ increased AP alone is not diagnostic, but an increase of both AP and inorganic phosphorus shows a statistically significant correlation. Although no statistically significant relationship to AP levels could be adduced in this study, there was a tendency towards increased values among $\mathrm{HO}$ cases, possibly because most of the patients observed suffered $\mathrm{HO}$ of grade 2 or higher severity. It has been suggested by others that the more marked the $\mathrm{HO}$, the more persistent the rise in AP levels. ${ }^{1}$ As all of our patients were receiving EHDP, inorganic phosphorus levels were consistently elevated.

\section{Conclusions}

A steady decline in radionuclide uptake was observed to extend anywhere from 3 months 
to 6 years beyond the time of radiographic maturation in 10 of 17 patients with $\mathrm{HO}$. Evidence of maturation as seen in bone scans occurred earlier than was seen in radiographs in 3 patients, and maturation appeared to be simultaneous in 4 . The evolution of $\mathrm{HO}$ was seen to take place over a period ranging between 3 and 80 months, for reasons that are not clear. Quantitative determinations of uptake ratios varied from a minimum of 1.10 to a maximum of 7.22 , with higher grades of $\mathrm{HO}$ corresponding to higher levels of uptake. We feel that, for grades of $\mathrm{HO}$ above 2, stabilization may be defined in terms of uptake ratios or 2.0 or less, provided that the initial uptake ratios are greater than 3.0. In cases in which initial uptake ratios are over 5.0, stabilization is indicated by uptake ratios of 3.0 or less. For grade 1 and grade $2 \mathrm{HO}$, however, these criteria may not apply. Finally, elevation of serum AP levels seems to occur only in association with the formation of $\mathrm{HO}$ above grade 2 .

\section{Acknowledgement}

This study was supported by a grant from the Department of Veterans Affairs.

\section{References}

1 Rossier AB, Bussat PH, Infante F et al (1973) Current facts on para-osteoarthropathy (POA). Paraplegia 11: $36-78$.

2 Stover SL (1986) Heterotopic ossification after spinal cord injury. In: Bloch RF and Basbaum M, editors. Management of Spinal Cord Injury. William and Wilkins, Baltimore: 284-301.

3 Orzel JA, Rudd TG (1985) Heterotopic bone formation: clinical, laboratory, and imaging correlation. $J$ Nucl Med 26: 125-132.

4 Pracash V (1983) Radionuclide assessment of heterotopic ossification in spinal cord injury patients. $J A m$ Paraplegia Soc 6: 10-12.

5 Tanaka T, Rossier AB, Hussey RW et al (1977) Quantitative assessment of para-osteo-arthropathy and its maturation in serial radionuclide bone images. Radiology 123: 217-221.

6 Morgenstern S, Kessler G, Auerbach J et al (1965) An automated p-nitrophenylphosphate serum alkaline phosphatase procedure for the AutoAnalyzer. Clin Chem 11: 876-888.

7 Mucheim G, Donath A, Rossier AB (1973) Serial scintigrams in the course of ectopic bone formation in paraplegic patients. Am J Roentgenol 118: 865-869.

8 Finerman GAM, Stover SL (1981) Heterotopic ossification following hip replacement or spinal cord injury. Two clinical studies with EHDP. Metab Bone Dis Relat Res 3: 337-342.

9 Stover SL, Hahn HR, Miller JM 3d (1976) Disodium etidronate in the prevention of heterotopic ossification following spinal cord injury (preliminary report). Paraplegia 14: 146-156.

10 Kim SW, Charter RA, Chai CJ et al (1990) Serum alkaline phosphatase and inorganic phosphorus values in spinal cord injury patients with heterotopic ossification. Paraplegia 28: 441-447. 